\title{
IMPACTOS ASSOCIADOS AO USO PROLONGADO DE BISFOSFONATOS NO TRATAMENTO DA OSTEOPOROSE EM IDOSOS
}

\author{
IMPACTS ASSOCIATED WITH PROLONGED USE OF BISPHOSPHONATE IN THE TREATMENT \\ OF OSTEOPOROSIS IN THE ELDERLY
}

\author{
Edilane Santos de Souza \\ Juliete de Jesus Santos \\ Lourenço Luis Botelho de Santana ${ }^{3}$
}

A osteoporose é uma das principais causas de morbidade e mortalidade em idosos no Brasil. Acomete cerca de 10 milhões de pessoas com prevalência de 11 a 23,8\% de fraturas ósseas, sendo considerada uma das maiores causas de internação hospitalar. Os bisfosfonatos (BFS) que atuam diretamente na remodelação óssea, são os fármacos de primeira escolha para o seu tratamento, porém o seu uso prolongado ocasiona danos severos. Este trabalho teve como objetivo identificar os impactos do uso prolongado de bisfosfonatos no tratamento da osteoporose em idosos. Foi realizada uma revisão de literatura através das bases de dados Lilacs, Scielo, Portal de Periódicos da CAPES e MEDLINE, através dos seguintes descritores: "osteoporose", "idosos" e "bisfosfonatos". A busca teve como critérios de inclusão publicações em língua portuguesa, inglesa ou espanhola, no período 2007 a 2018. Os trabalhos mostram que os bisfosfonatos têm sua eficácia e segurança comprovada na redução de fraturas vertebrais, não vertebrais e femorais em pacientes com osteoporose, porém seu uso prolongado pode causar osteonecrose maxilar e fraturas atípicas no fêmur. Com isso, percebe-se que no tratamento da osteoporose é importante acompanhar os pacientes que fazem uso de bisfosfonatos por longo período, no intuito de evitar a ocorrência destas fraturas.

Palavras-chave: Bisfosfonatos. Idoso. Osteoporose.

Osteoporosis is one of the main causes of morbidity and mortality in elderly people in Brazil. It affects approximately 10 million people with a prevalence of 11 to $23.8 \%$ of bone fractures, being considered one of the major causes of hospitalization. Bisphosphonates that act directly on bone remodeling are the first choice drugs for its treatment, but their prolonged use may cause severe damage. This study aimed to identify the impacts of prolonged use of bisphosphonates in the treatment of osteoporosis in the elderly. The databases Lilacs, Scielo, Portal de Periódicos CAPES and MEDLINE were searched to collect the data for this literature review using the following descriptors: "osteoporosis", "elderly" and "bisphosphonates". The inclusion criteria were publications in Portuguese, English or Spanish published between 2007 and 2018. The studies report that bisphosphonates are efficient and save to reduce vertebral, non-vertebral and femoral fractures in patients with osteoporosis, but their prolonged use may cause maxillary osteonecrosis and atypical fractures in the femur. Thus, it is suggested that the treatment of osteoporosis requires the close follow-up of patients who use bisphosphonates for a long period in order to avoid the occurrence of abnormal fractures.

KEYWORDS: Bisphosphonate. Elderly. Osteoporosis.

\footnotetext{
${ }^{1}$ Graduada em Farmácia- Centro Universitário Estácio da Bahia/Estácio-FIB. Salvador, Bahia; http://lattes.cnpq.br/9478732308078466. Email: ninoka.saj@hotmail.com.

Graduada em Saúde- Universidade Federal do Recôncavo da Bahia (UFRB), Centro de Ciências da Saúde. Santo Antônio de Jesus,

Bahia; http://lattes.cnpq.br/9510260460909113. Email: jusantos.21@hotmail.com.

${ }^{3}$ Doutorado em Química- Universidade do Estado da Bahia(UNEB), Salvador, Bahia; http://lattes.cnpq.br/6607021567646943. Email:

Ilsantana@uneb.br
} 


\section{INTRODUÇÃO}

A osteoporose é uma doença que afeta mundialmente cerca de $7 \%$ dos homens e $17 \%$ das mulheres, sendo que $70 \%$ dos indivíduos acometidos encontram-se na terceira idade. No Brasil, 10 milhões de pessoas sofrem de osteoporose, tendo como agravante a prevalência de $11 \%$ a $23,8 \%$ de fraturas relacionadas à fragilidade óssea, sendo assim considerada uma das maiores causas de internação hospitalar, gerando altos custos para o Sistema Único de Saúde (SUS) (MORAES, 2014).

Com o envelhecimento, as alterações anatômicas e fisiológicas tornam os idosos mais frágeis e, ao mesmo tempo, limitados na sua capacidade de realizar atividades do cotidiano (SMITH; SHAH, 2018). A osteoporose é caracterizada pela redução de massa óssea, que ocasiona fraturas com facilidade e é considerada também um problema de saúde pública, sendo uma das principais causas de morbidade e mortalidade em idosos. Esta redução está associada a um conjunto de fatores, como a menopausa, o envelhecimento, hereditariedade, dieta pobre em cálcio, excesso de fumo e álcool, falta de atividade física e uso de alguns medicamentos (LEITE, 2014; SMITH; SHAH, 2018).

Entre os fármacos utilizados no tratamento da osteoporose destacam-se os bisfosfonatos (alendronato, ibandronatro, ácido zoledrônico, risedronato, pamidronato), que são empregados nos diversos tipos da doença como na osteoporose senil, pós-menopáusica e a induzida por uso de corticoides. O uso destes fármacos tem como função prevenir as fraturas através do aumento da massa óssea (BRATS, 2013).

Os bisfosfonatos (BFS) podem ser administrados por via oral ou injetável; no entanto, o uso oral é considerado a primeira escolha para o tratamento da osteoporose. Embora por via oral apenas uma pequena parte do fármaco administrado está associado ao tecido ósseo, a eficácia deste modo de administração foi comprovada na redução de fraturas e no aumento da densidade mineral óssea. $O$ grande problema é que o uso durante períodos prolongados pode provocar efeitos adversos como osteonecrose da mandíbula e fratura atípica do fêmur (CAIRES, 2017; MOTA, 2012).

Dessa forma, este estudo teve como objetivo identificar os impactos do uso prolongado de bisfosfonatos no tratamento da osteoporose em idosos.

\section{METODOLOGIA}

Esta revisão bibliográfica sobre o tratamento com bisfosfonatos de idosos portadores de osteoporose tem caráter exploratório, descritivo e qualitativo. A busca dos artigos foi realizada nas seguintes bases de dados: Literatura Latino-Americana e do Caribe em Ciências da Saúde (LILACS), Scientific Eletronic Library Online (SCIELO), Portal de Periódicos da Coordenação de Aperfeiçoamento de Pessoal de Nível Superior (CAPES) e MEDLINE. Utilizaram-se os seguintes descritores: "osteoporose", "idosos" e "bisfosfonatos". A busca seletiva dos trabalhos científicos teve como critérios de inclusão: artigos científicos, teses e dissertações em língua portuguesa, inglesa ou espanhola, publicados durante o período 2007 a 2018. Inicialmente foram selecionados 98 (noventa e oito) publicações, das quais 46 (quarenta e seis) foram excluídas por apresentarem problemas metodológicos ou não se adequarem ao tema. Sendo assim, na revisão foram utilizadas 52 publicações adequadas aos critérios adotados para o estudo e aos objetivos propostos.

\section{RESULTADOSE DISCUSSÃO}

\section{Osteoporose}

A osteoporose é caracterizada como uma doença osteometabólica que é resultado da redução da massa óssea total, quando a velocidade da reabsorção do osso é maior que a velocidade de formação, 
tornando assim os ossos porosos e quebradiços ocasionando aumento de fraturas, deformidades e dores crônicas, cuja consequência é, em última instância, o aumento da mortalidade. Ao longo da vida, estima-se que $50 \%$ das mulheres e $20 \%$ dos homens acima de 50 anos provavelmente terão fratura osteoporótica. Destes, 5\% morrem durante a internação hospitalar com fraturas de quadril, 12\% três meses depois, e 20\% um ano após a fratura (MINISTÉRIO DA SAÚDE, 2014; SILVA, 2015).

Em um estudo realizado pelo Boletim Brasileiro de Avaliação de Tecnologias em Saúde (BRATS, 2013), identificou-se que, no ano de 2009, o número de internações hospitalares decorrentes de fraturas osteoporóticas em mulheres foi de 20.778 mil e em homens de 10.020 mil, mostrando assim que o número de mulheres internadas é duas vezes maior do que o de homens. Os gastos com internações hospitalares foram de $\mathrm{R} \$ 57,61$ milhões, e os referentes a medicamentos foram de 24,77 milhões. Por sua vez, Moraes et al., (2014) identificaram que o número de procedimentos realizados no Brasil entre 2008 e 2010 relacionados com osteoporose em idosos foi de 3.252 .756 , totalizando assim um custo de $\mathrm{R} \$ 288.986 .335,15$. Deste modo, ficam evidenciados os altos gastos do SUS com fraturas provocadas pela osteoporose em idosos (MORAES, 2014).

Segundo o estudo The Brazilian Osteoporosis Study (BRAZOS, 2006), realizado nas cinco regiões brasileiras, com 2.420 indivíduos (725 homens e 1695 mulheres com idade acima de 40 anos), o número de fraturas causadas por baixo impacto foi de $73,2 \%$ no sexo masculino e $85 \%$ no sexo feminino (PINHEIRO, 2010).

Os principais fatores de risco desta doença são: a menopausa, o envelhecimento, histórico familiar de osteoporose, ingestão deficiente de cálcio, deficiência de vitamina $D$, tabagismo, alcoolismo, sedentarismo, uso de alguns medicamentos como hormônios tireoidianos, anticonvulsivantes e corticoides, diabetes, doenças reumáticas e doenças renais, além da falta de um estilo de vida saudável (SOUZA, 2013; GUERRA, 2010).

Além disso, a osteoporose é dez vezes mais comum nas mulheres do que nos homens, especialmente na pós-menopausa, por consequência da diminuição de estrogênio, que resulta numa perda de massa óssea mais rápida. A formação da massa óssea ocorre desde a infância até os 35 anos, quando chega ao seu pico máximo, sendo que após essa fase ocorre gradualmente uma perda de entre $0,3 \%$ a $0,5 \%$ ao ano. Em mulheres na menopausa, a perda é 1,5\% aproximadamente, podendo aumentar para $3 \%$ a $4 \%$ por ano (FERNANDES, 2016; ANDRADE, 2015).

Os bisfosfonatos são frequentemente prescritos para essa patologia, por terem alto grau de segurança e geralmente serem bem tolerados; porém, o seu uso prolongado está relacionado com efeitos colaterais indesejados como a osteonecrose maxilar e fraturas atípicas na região do fêmur (ARANTES, 2010).

\section{Bisfosfonatos (BFS)}

Os bisfosfonatos, compostos análogos ao pirofosfato (Figura 1), foram sintetizados pela primeira vez em 1865 e utilizados na indústria como agentes anticorrosivos. Seu uso no tratamento de doenças ósseas iniciou-se após estudos realizados in vitro e in vivo que comprovavam seu efeito no controle da formação e dissolução do fosfato na mineração e reabsorção óssea (SAMPAIO et al., 2010; CARDONA et al., 2009).

Figura 1. Estrutura do pirofosfato e estrutura geral dos bisfosfonatos.

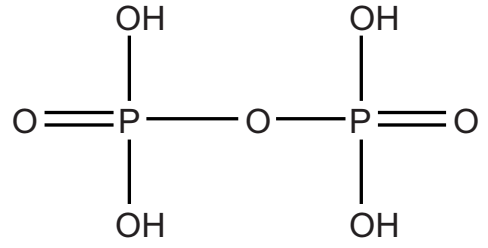

Pirofosfato<smiles>[R]C([R2])(P(=O)(O)O)P(=O)(O)O</smiles>

Bisfosfonato

Fonte: CARDONA et al., 2009. 
Por sofrer uma rápida hidrólise enzimática, o pirofosfato não é utilizado em tratamento de doenças ósseas; já o bisfosfonato, devido à presença de um átomo de carbono central, apresenta resistência à degradação enzimática, meia-vida biológica mais longa e capacidade de atuar no metabolismo ósseo. Além disso, os diferentes radicais $\left(R_{1}\right.$ e $\left.R_{2}\right)$ ligados ao carbono central no bisfosfonato possibilitam uma diversidade de análogos estruturais com diferentes afinidades pelos cristais ósseos e consequentemente diferentes intensidades de ação (Figura 1) (GEORGE et al., 2018).

Os bisfosfonatos orais possuem pouca absorção pelo intestino (1\% a 7\%), sofrem eliminação renal e são absorvidos por meio da ligação ao cálcio nos cristais de hidroxiapatita, possuindo alta afinidade pela matriz óssea. Dentre os bisfosfonatos, o ácido zoledrônico é o que tem maior afinidade e liga-se rapidamente à matriz óssea; porém, sua distribuição pelo osso é lenta, enquanto que o clodronato, com menor afinidade, tem uma distribuição mais ampla e rápida (PASSERI et al., 2011; KHAJURIA et al., 2011). Diante disso, os bisfosfonatos são administrados com o intuito de melhorar a estrutura óssea, uma vez que inibem a dissolução de hidroxiapatita e promovem a apoptose dos osteoclastos, desacelerando a reabsorção, o que diminui a perda óssea e consequentemente as fraturas (GUPTA, 2016).

Desta maneira, os bisfosfonatos, ao bloquear os osteoclastos, diminuem a velocidade de reabsorção óssea, reduzem a perda e recuperam a densidade mineral óssea, promovendo a redução de fraturas. Os fármacos orais ou intravenosos possuem o mesmo grau de eficácia; os orais mais utilizados no tratamento da osteoporose são o alendronato e risedronato devido ao baixo custo e a simplicidade do uso (CARVALHO et al., 2010).

A primeira geração de bisfosfonato surgiu no ano de 1977, com a produção do etidronato. A partir de então, novas gerações foram sendo desenvolvidas, como o alendronato na segunda geração, que surgiu no ano de 1991, e o ácido zoledrônico da terceira geração, em 2001. A cada geração observa-se um aumento de 10 vezes nas propriedades antirreabsortivas destes bisfosfonatos (POUBEL et al., 2012).

As propriedades antirreabsortivas dos bisfosfonatos são divididas por gerações, o que confere aos fármacos desta classe a capacidade de possuir diferentes potências. O etidronato, clodronato e tiludronato fazem parte da primeira geração, o alendronato e o pamidronato da segunda geração e na terceira geração estão inclusos o risedronato, ibandronato e o zoledronato (BARIN et al., 2016; NUNES et al., 2010) Os bisfosfonatos podem ainda ser classificados de acordo com a presença ou ausência de nitrogênio no grupo $R_{2}$, cuja presença confere maior potência à droga (Tabela 1) (SHARMA et al., 2013).

Os dados apresentados na Tabela 1 permitem a identificação das características dos bisfosfonatos, sendo analisados os seguintes aspectos: os tipos de bisfosfonatos, potência, geração, categoria, via de administração e indicação.

Tabela 1. Características dos bisfosfonatos

\begin{tabular}{cccccc}
\hline Bisfosfonatos & Potência & Geração & Categoria & $\begin{array}{c}\text { Via de } \\
\text { Administração }\end{array}$ & Indicação \\
\hline Etidronato & $1 \mathrm{x}$ & $1^{\mathrm{a}}$ & Não Nitrogenado & Oral Intravenosa & Paget Osteoporose \\
\hline Tiludronato & $10 \mathrm{x}$ & $1^{\mathrm{a}}$ & Não Nitrogenado & Oral & Neoplasia \\
\hline Clodronato & $10 \mathrm{x}$ & $1^{\mathrm{a}}$ & Não Nitrogenado & Oral Intravenosa & Doenças Malignas \\
\hline Pamidronato & $100 \mathrm{x}$ & $2^{\mathrm{a}}$ & Nitrogenado & Endovenosa & Paget Neoplasia \\
Alendronato & $500 \mathrm{x}$ & $2^{\mathrm{a}}$ & Nitrogenado & Oral & Osteoporose Paget \\
\hline Ibandronato & $1000 \mathrm{x}$ & $3^{\mathrm{a}}$ & Nitrogenado & Oral Intravenosa & Osteoporose \\
Risedronato & $2000 \mathrm{x}$ & $3^{\mathrm{a}}$ & Nitrogenado & Oral & Osteoporose \\
Ácido & $10000 \mathrm{x}$ & $3^{\mathrm{a}}$ & Nitrogenado & Endovenosa & $\begin{array}{c}\text { Paget Neoplasia } \\
\text { Zoledrônico }\end{array}$ \\
\hline
\end{tabular}

Fonte:(NUNES et al. 2010; CARVALHO et al., 2010; BARIN et al., 2016; TORREGROSA; RAMO, 2010). 
A Tabela 1 mostra que os bisfosfonatos, além de serem usados na terapia da osteoporose, são também empregados no tratamento de outras doenças como neoplasias malignas, doenças de Paget, hipercalcemia e mieloma múltiplo (IZQUIERDO et al., 2011).

Quando analisadas as características dos BFS, observa-se que o clodronato, tiludronato e etidronato não possuem nitrogênio em sua estrutura química e são classificados como de primeira geração, sendo o etidronato o que possui indicação para o tratamento da osteoporose. $\mathrm{O}$ alendronato e pamidronato são nitrogenados e considerados de segunda geração, mas apenas o alendronato possui indicação na terapia da osteoporose. Já o risedronato, ácido zoledrônico e ibandronato são BFS de terceira geração que possuem nitrogênio na sua estrutura, são indicados para terapia de osteoporose e são os que possuem potência maior comparados com os de primeira e segunda geração (NUNES et al. 2010; CARVALHO et al., 2010; BARIN et al., 2016; TORREGROSA; RAMO, 2010). O estudo realizado por Coelho, Gomes e Fernandes (2010) mostra que o etidronato, tiludronato, alendronato, risendronato e ibandronato são os bisfosfonatos de administração oral mais indicados para a terapia da osteoporose. Os bisfosfonatos citados acima são registrados no Brasil, entretanto foram incorporados pelo Sistema Único de Saúde (SUS) apenas o alendronato, risedronato e pamidronato (COELHO et al., 2010).

Três meses após o início da terapia oral com os bisfosfonatos, nota-se supressão da reabsorção óssea e quando a terapia é endovenosa a ação é mais rápida. Entretanto, a literatura relata que após três anos de tratamento com os bisfosfonatos, a densidade mineral óssea da coluna vertebral aumenta em 5$8 \%$ e do quadril em 3-6\% (KHAJURIA et al., 2011).

Diante do exposto, percebe-se que os medicamentos à base de bisfosfonatos são os mais prescritos e considerados de primeira escolha para o tratamento da osteoporose, devido ao seu efeito na reabsorção óssea, proporcionando assim uma melhora na qualidade de vida para os pacientes que sofrem com dor ou eventos indesejados desta doença.

\section{Impactos do Uso dos Bisfosfonatos no Tratamento da Osteoporose}

Como já visto, a osteoporose é uma doença crônica e os bisfosfonatos são um dos fármacos de primeira escolha utilizados para seu tratamento, que geralmente se estende por toda a vida do paciente. Apesar de sua eficiência, a literatura descreve alguns efeitos adversos relacionados ao uso prolongado da substância, sendo os principais a osteonecrose da mandíbula e fratura femoral atípica (Tabela 2) (SIRIS et al., 2011; ALI et al., 2009).

Tabela 2. Perfil de impactos associados com o uso de bisfosfonatos.

\begin{tabular}{|c|c|c|c|}
\hline Referência & Bisfosfonato & Tempo de uso & Impactos \\
\hline RUGGIERO et al.,2009 & Alendronato & Mais de três anos & Osteonecrose Maxilar \\
\hline COELHO et al., 2010 & BFs orais & $\begin{array}{c}\text { Período Longo Iguais ou } \\
\text { Superiores a } 3 \text { Anos }\end{array}$ & $\begin{array}{l}\text { Necrose óssea } \\
\text { dos maxilares }\end{array}$ \\
\hline OTERO et al., 2011 & $\begin{array}{l}\text { Ácido Zoledrônico } \\
\text { Alendronato }\end{array}$ & $\begin{array}{c}1 \text { ano e } 7 \text { meses } \\
3 \text { anos }\end{array}$ & $\begin{array}{l}\text { Necrose óssea } \\
\text { dos maxilares }\end{array}$ \\
\hline DUPLAT et al., 2016 & $\begin{array}{c}\text { Aminobisfosfonatos } \\
\text { por Via Oral }\end{array}$ & Acima de 3 Anos & $\begin{array}{l}\text { Osteonecroses } \\
\text { Maxilares }\end{array}$ \\
\hline MILANI et al., 2012 & Ácido Zoledrônico & 1 ano & Osteonecrose Maxilar \\
\hline PRETTO et al., 2014 & Alendronato & 3 a 5 anos & $\begin{array}{c}\text { Fratura Atípica do } \\
\text { Fêmur }\end{array}$ \\
\hline
\end{tabular}




\begin{tabular}{cccc}
\hline BRATS, 2013: & $\begin{array}{c}\text { Alendronato } \\
\text { lbandronato } \\
\text { Ácido Zoledrônico }\end{array}$ & Mais de 3 anos & $\begin{array}{c}\text { Fraturas Atípicas e } \\
\text { Eventos Inflamatórios } \\
\text { da mandíbula }\end{array}$ \\
MOURÃO et al., 2013 & BFs intravenosos & 3 anos & Osteonecrose Maxilar \\
RUGGIERO et al., 2014 & BFs orais & Mais de 4 anos & $\begin{array}{c}\text { Osteonecrose } \\
\text { Maxilar }\end{array}$ \\
KATZ; ORDEVA, 2014 & Ácido Zoledrônico & 1 ano & Osteonecrose Maxilar \\
PRETTO et al., 2014 & BFs orais & Mais de 3 anos & Osteonecrose Maxilar \\
TEMPONI et al., 2015 & BFs oral & 6 anos & Fratura Atípica do \\
Fêmur
\end{tabular}

Avaliando os trabalhos destacados na Tabela 2, verifica-se que, dentre os impactos relacionados com o uso prolongado de bisfosfonatos, aproximadamente $81,25 \%$ estão relacionados com osteonecrose maxilar e 18,75\% com fraturas atípicas no fêmur. A osteonecrose que acontece no maxilar é identificada pela exposição do osso necrótico na região maxilofacial que segue mais de oito semanas sem cicatrização sem nenhum histórico de radioterapia na região maxilofacial (COELHO et al., 2010). De acordo com Izquierdo, Oliveira e Weber (2011), a osteonecrose associada aos bisfosfonatos restringe-se aos ossos maxilomandibulares, comprometendo a mandíbula. Considera-se como fatores relevantes para o surgimento destes efeitos colaterais o bisfosfonato utilizado, a via de administração e a duração do tratamento, sendo o risco maior oferecido pelos bisfosfonatos nitrogenados com administração endovenosa (IZQUIERDO et al., 2011).

Os primeiros casos de osteonecrose maxilar foram diagnosticados e relatados no ano de 2003 . A osteonecrose causada pelo uso do bisfosfonato pode levar um tempo sem apresentar sintomas, embora também possa haver reações como dor, inchaço maxilar, afrouxamento dos dentes, dificuldade de se alimentar e de falar (FICARRA; BENINATI, 2007). Essa patologia costuma-se apresentar após procedimentos dentários e, com menor frequência, de forma espontânea. A seletividade da região de impacto está associada ao fato dos bisfosfonatos apresentarem concentrações maiores na região óssea mandibular e maxilar comparado a outros ossos (RUGGIERO, 2007).

A osteonecrose decorrente do uso de bisfosfonatos está relacionada com a intensa inibição dos osteoclastos, pois após a apoptose, os mesmos não são substituídos e o sistema capilar ósseo é comprometido. A osteonecrose pode ser classificada por estágios: no estágio I ocorre a exposição do osso em pacientes que utilizam medicamentos, mas não apresentam sintomas ou infecção; no estágio II ocorre a exposição do osso associada a sintomas como dor e infecção; e no estágio III há exposição do osso com quadro sintomático infeccioso associado a outras alterações, como fratura patológica (ROSEMONT, 2007).

Dessa forma, o tratamento da osteonecrose com bisfosfonatos depende do estágio da doença: no estágio I será suspenso o uso do bisfosfonato e é indicado usar o enxaguamento bucal de clorexidina a $0,12 \%$ três vezes ao dia por um período de 15 dias; no estágio II indica-se usar antibióticos (amoxicilina + clavulanato) a cada 12 horas durante 15 dias em combinação com anti-inflamatórios não esteroidais. Já no estágio III utiliza-se a associação do tratamento do estágio I e II, acrescido da retirada do tecido morto 
(REYNA; PEREZ, 2015).

Assim, a utilização de bisfosfonatos está associada com várias reações adversas como dores ósseas, musculares, articulares e com maior frequência a osteonecrose maxilar ou osteonecrose, sendo considerada uma séria reação adversa, cujo mecanismo ainda não é conhecido (SAMPAIO et al., 2010).

Outro evento relacionado ao uso prolongado de bisfosfonatos orais são as fraturas atípicas do fêmur, associadas principalmente a tratamentos da osteoporose com duração de mais de três anos (GIORDANO et al., 2013). O uso prolongado de alendronato de sódio está relacionado com fraturas atípicas do fêmur (como especificado na sua bula). Esse tipo de fratura corresponde a $1 \%$ das fraturas femorais que podem levar um longo tempo para cura. Acredita-se que essas fraturas atípicas aconteçam em decorrência da supressão excessiva e prolongada da remodelação óssea, causando um dano na função mecânica que leva a microfraturas e à fragilidade nos ossos. Sendo assim, alguns especialistas sugerem um período de suspensão do uso dos bisfosfonatos, pois o aparecimento da osteonecrose maxilar e fraturas atípicas do fêmur estão relacionados ao uso prolongado e, conforme a literatura, não foi comprovada a eficácia desses fármacos após cinco anos de terapia (CAIRES et al., 2017; RADOMINSKI et al., 2017).

Diante dos resultados apresentados neste estudo e no que foi visto na literatura, percebe-se que os bisfosfonatos são bastante utilizados no tratamento da osteoporose pela sua eficácia e segurança comprovada na redução de fraturas vertebrais, não vertebrais e femorais em idosos. Porém, vale ressaltar que seu uso prolongado pode acarretar sérias reações adversas como a osteonecrose maxilar e fraturas atípicas no fêmur. Os bisfosfonatos envolvidos nessa reação são, principalmente, os de via endovenosa; entretanto, estudos comprovam que os de administração oral também podem causar, em alguns casos, esse tipo de efeito colateral. Sendo assim, é importante acompanhar os pacientes que fazem uso de bisfosfonatos há mais de três anos para interrupção do tratamento caso sejam observados estes eventos adversos.

\section{CONSIDERAÇÕES FINAIS}

Entende-se que a osteoporose é uma doença silenciosa com prevalência na terceira idade, principalmente no sexo feminino, e está relacionada também com fatores de risco associados ao estilo de vida. É um problema de saúde pública, devido aos altos custos com internações hospitalares ocasionadas pelas fraturas que podem causar morbidade e mortalidade.

Os fármacos de primeira escolha para essa doença são os bisfosfonatos, que atuam diretamente na remodelação óssea com eficácia e segurança. Entretanto, deve-se ter cuidado com o uso prolongado desses fármacos, pois acima de três anos de uso, os estudos comprovaram complicações gravíssimas prevalentes como osteonecrose maxilar e fraturas atípicas no fêmur.

\section{REFERÊNCIAS}

AIEX, L.S.; JIMENEZ, M.V.; MILENA, A.P. Osteonecrose mandibular relacionada com bisfosfonatos orais em paciente idosa polimedicada. Revista Brasileira de Medicina de Família e Comunidade, v. 10, n.36, p. 1-7, 2015.

ALI, T.; JAY, R.H. Spontaneous femoral shaft fracture after long-term alendronate. Age and Ageing, v. 38, n.1, p. 625-626, 2009.

ANDRADE, S.A.F. Osteoporose: um problema de saúde pública. Revista Unilus Ensino e Pesquisa, v. 28, n.12, p. 41-47, 2015.

ARANTES, H.P.; SILVA, A.G.; CASTRO, M.L. Bisphosphonates in the treatment of metabolic bone diseases. \&Arquivos Brasileiro de Endocrinologia Metabologia, v. 54, n.2, p.206-212, 2010. 
BARIN, L.M.; PILLUSKY, F.M.; PASINI, M.M.; DANESI, C.C. Osteonecrose dos Maxilares Associada ao Uso de Bisfosfonatos: Uma Revisão De Literatura. Revista de Odontologia da Universidade de São Paulo, v. 28, n.2, p. 126-134, 2016.

BOLETIM BRASILEIRO DE AVALIAÇÃO DE TECNOLOGIAS EM SAÚDE - BRATS. Eficácia e segurança do uso dos Bisfosfonatos por longo prazo para prevenção de fraturas osteoporóticas em mulheres na pós-menopausa, v. 2, n. 21, p.1-14, 2013.

BRASIL. Ministério da Saúde. Portal da Saúde. Portaria SAS/MS n 451. Protocolo Clínico e Diretrizes Terapêutica da Osteoporose. Brasília, DF, 26 de março de 2014 . Disponível em http://portalarquivos.saude.gov.br/images/pdf/2014/abril/02/pcdt-osteoporose-2014.pdf. Acesso em 19/12/2017

CAIRES, E.L.P; BEZERRA, M.C., JUNQUEIRA, A.F.T.A., FONTENELE, S.M.A, ANDRADE, S.C.A; D'ALVA, C.B. Revista Brasileira de Reumatologia, v.3, n.57, p.254-263, 2017.

CARDONA, F.; BAGÁN, J.V.; SÁINZ, E.; FIGUERIDO, J.; GINE, F.; VIDÁ, F.J. Osteonecrosis de los maxilares por bisfosfonatos: Actualización y puesta al día. Anales del Sistema Sanitario de Navarra, v. 32, n.3, p. 423-437, 2009.

CARVALHO, P.S.; SANTOS, H.F.; DUARTE, B.G.; CARVALHO, F.A.; RIBEIRO, E.D.; ROCHA, J.F. Principais aspectos da cirurgia bucomaxilofacial no paciente sob terapia com bisfosfonatos. Revista da Faculdade de Odontologia Universidade de Passo Fundo, v. 15, n. 2, p. 183-189, 2010.

COELHO, A.I.; GOMES, P.S.; FERNANDE, M.H. Osteonecrose dos maxilares associada ao uso de bisfosfonatos. Parte II: Linhas de Orientação na Consulta de Medicina Dentária. Revista Portuguesa de Estomatologia, Medicina Dentária e Cirurgia Maxilofacial, v. 51, n.3, p. 185-191, 2010.

DUPLAT C.B.; COSTA, D.D.; FALCÃO, A.F.P. Osteonecrose em Maxilares Induzida Por Bisfosfonatos: Farmacologia e Condutas Clínicas. Revista Saúde, v.8, n.2, p. 69-78, 2016.

FERNANDES, T.R.L.; OLIVEIRA, J.B.; LORENCETE, T.V.; AMADEI, J.L. Fatores associados à osteoporose em mulheres na pós-menopausa. Semina: Ciências Biológicas e da Saúde, v. 36, n.1, p. 93-106, 2015.

GEORGE, E.L.; LIN, Y.L.; SAUNDERS, M. M. Bisphosphonate-related osteonecrosis of the jaw: a mechanobiology perspective. Bone Reports, v.8, p.104-109, 2018.

GIORDANO, V.; LAGES, M.M.; SANTANA, E.; SOUZA F.S.; ALBUQUERQUE, R.P.; AMARAL, N.P. Fraturas femorais atípicas por uso prolongado de bisfosfonatos Mitos e verdades. Jornal Brasileiro de Medicina, v. 101, n.2, p. 13-17, 2013.

GUERRA, M.T.; PRADO, G.L. Osteoporose em mulheres na pós-menopausa: perfil epidemiológico e fatores de risco. Revista Brasileira de Clínica Médica, v. 5, n. 8, p. 386-391, 2010.

GUPTA, A.; MARCH, L. Treating osteoporosis. Australian Prescriber, v. 39, n.2, p. 40-46, 2016.

GUTIÉRREZA, L.Q.; GUTIÉRREZB, I.Q.; GALINDOC, A.M.; GARCÍA, M.J.C., ZAMORANO, M.J, TOBOSO, J.B. Osteonecrosis mandibular secundaria a bisfosfonato. Medicina General y de Familia, v. 5, n. 1, p. 33-55, 2016.

FICARRA, G.; BENINATI, F. Bisphosphonate-related Osteonecrosis of the Jaws: An Update on Clinical, Pathological and ManagementAspects. Head and neck pathology, v.1, n.1, p. 132-140, 2007.

IZQUIERDO, C.M.; OLIVEIRA, M.G.; WEBER, J.B.B. Terapêutica com bisfosfonatos: implicações no paciente odontológico. Revista da Faculdade de Odontologia Universidade de Passo Fundo, v. 16, n.3, p. 347-352, 2011.

KATZ, J.; ORDOVEZA, P.A. Bisphosphonate-related Osteonecrosis of the jaw (BRONJ) associated with a once- 
yearly IV infusion of zoledronic acid (Reclast) $5 \mathrm{mg}$ : two cases and review of the literature. Quintessence International, v. 45, n.8, p. 685-690, 2014.

KHAJURIA, D.K.; RAZDAN, R.; MAHAPATRA, D.R. Drugs for the management of osteoporosis: a review. Revista Brasileira de Reumatologia, v.51, n.4, p. 365-382, 2011.

LEITE, S.C.; BARATTO, I.; SILVA, R. Consumo de Cálcio e risco de osteoporose em um grupo de idosos de Guarapuava. Revista Brasileira de Obesidade, Nutrição e Emagrecimento, v. 8, n.48, p. 165-174, 2014.

MILANI, C.M.; LOBO, M.; CARRILHO, E.; SOUZA, J.A; MACHADO, M.A. Biphosphonate related osteonecrosis of the jaw: case report. Revista Odonto, v. 39, 20, p. 27-33, 2012.

MORAES, L.F.S; SILVA, E.M; SILVA, D.A.S.; PAULA, A.P. Gastos com o tratamento da osteoporose em idosos do Brasil (2008-2010): análise dos fatores associados. Revista Brasileira de Epidemiologia, v.1, n.1, p.719, 2014.

MOTA, LS; SOUSA, E.G; AZEVEDO, F.H.C. Intercorrências da osteoporose na qualidade de vida dos idosos. Revista Interdisciplinar NOVAFAPI, v.5, n.2, p. 44-49, 2012.

MOURÃO, C.F.; MOURA, A.P.; MANSO, J.E.F. Tratamento da osteonecrose dos maxilares associada aos bisfosfonatos: revisão da literatura. Revista Brasileira de Cirurgia de Cabeça e Pescoço, v. 42, n.2, p. 113-117, 2013.

NUNES, V.; LOPES, B.; LORDANI, R.X.F.; ALVES, J.; ROCHA, R.; MACHADO, W.; OLIVEIRA, L.C. Uso de Bisfosfonatos em Pacientes com Câncer e sua Associação Com Osteonecrose dos Ossos Maxilares. Revista Periodontia, v. 20, n.3, p. 20-27, 2010.

OTERO J.J.; MAYORDOMO, A.R.; SUANZES J.C.; VILLAR, E.L.; ROSADO, R.L.; MOLINÍ, M.S.; BERART, P. Osteonecrosis de los maxilares asociada al uso de bisfosfonatos: revisión de ocho casos. Revista Española de Cirurgía oral y Maxilofacial, v. 33, n.1, p. 15-21, 2011.

PASSERI, A.L.; BÉRTOLO, M.B.; ABUABARA, A. Osteonecrose dos maxilares associada ao uso de bisfosfonatos. Revista Brasileira de Reumatologia, v. 51, n.4, p. 401-407, 2011.

PINHEIRO, M.M.; CICONELLI, M.R.; JACQUES, O.N.; GENARO, S.P.; MARTINI, A.L.; FERRAZI, B.M. O impacto da osteoporose no Brasil: dados regionais das fraturas em homens e mulheres adultos - The Brazilian Osteoporosis Study (BRAZOS). Revista Brasileira de Reumatologia, v. 50, n.2, p. 113-127, 2010.

POUBEL, V.L.N.; CRUZ, D.S.M.; GIL, L.F.; JÚNIOR, N.; CLAUS, J.D.; GIL, J.N. Osteonecrose maxilo-mandibular induzida por bisfosfonato: revisão bibliográfica. Revista de cirurgia e Traumatologia Buco-Maxilo-Facial, v. 12, n.1, p. 33-42, 2012.

PRETTO E.F.; PACHECO, M.; MORENO, A.; MESSA, O.; GNECCO, J. Bisphosphonate-induced osteonecrosis of the jaws: clinical,imaging, and histopathology findings. Oral surgery, oral medicine, oral pathology and oral radiology, v. 118, n.4, p. 408-417, 2014.

RADOMINSKI, S.C; BERNARDO, W.; PAULA, A.P.; ALBERGARIA, B.; MOREIRA, C.; FERNANDES, C.E.; CASTRO, C.H.M., ZERBINI, C.A.F.; DOMICIANO, D.S.; MENDONCA, L.M.C.; POMPEI, L.M.; BEZERRA, M.C.; LOURES, M.A.R.; WENDER, M.C.O.; CASTRO, M.L.; PEREIRA, R.M.R.; MAEDA, S.S.; SZEJNFELD, V.L.; BORBA, V.Z.C. Diretrizes brasileiras para o diagnóstico e tratamento da osteoporose em mulheres na pósmenopausa. Revista Brasileira de Reumatologia, v. 57, n.2, p. 452-456, 2017.

REYNA, J.H; PEREZ, J. R.A. Papel de los bisfosfonatos en la Osteonecrosis mandibular. Perinatología y Reproducción Humana, v.29, n.1, p.26-29, 2015. 
ROSEMONT, I.L. American Association of Oral and Maxillofacial Surgeons position paper on bisphosphonaterelated osteonecrosis of the jaws. Journal of oral and maxillofacial surgery, v.65, n.3, p. 369-376, 2007.

RUGGIERO, S.L.; DODSON, T.B.; ASSAEL, L.A.; LANDESBERG, R.; MARX, R.E.; MEHROTRA, B. American Association of Oral and Maxillofacial Surgeons Position Paper on Bisphosphonate-Related Osteonecrosis of the Jaws-2009 Update. Australian Endodontic Journal, v. 67, n.1, p. 2-12, 2009.

RUGGIERO, S.L.; DODSON, T.B.; FANTASIA, J.; GOODDAY, R.; AGHALOO, T. Mehrotra, B.; Ryan, F. American Association of Oral and Maxillofacial Surgeons Position Paper on Medication-Related Osteonecrosis of the Jaw-2014 Update. Journal of oral and maxillofacial surgery, v. 72, n.1, p. 1938-1956, 2014.

RUGGIERO, S.L. Guidelines for the diagnosis of bisphosphonate-related osteonecrosis of the jaw (BRONJ). Clinical cases in mineral and bone metabolism, v.4, n.1, p. 37-42, 2007.

SAMPAIO, F.C., VELOSO, H.H.P.; BARBOSA, D.N. Mecanismos de ação dos bisfosfonatos e sua influência no prognóstico do tratamento endodôntico. Revista da Faculdade de Odontologia de Porto Alegre, v.51, n.1, p. 31 $38,2010$.

SHARMA, D.; IVANOVSKI, S.; SLEVIN, M.; HAMLET, S.; POP, T.S.; BRINZANIUC, K.; PETCU, E.B.; MIROIU, R. Bisphosphonate-related osteonecrosis of jaw (BRONJ): diagnostic criteria and possible pathogenic mechanisms of an unexpected anti-angiogenic side effect. Vascular Cell, v.5, n.1, p.2-8, 2013.

SIRIS, E.S.; PASQUALE, M.K.; WANG, Y.; WATTS, N.B. Estimating bisphosphonate use and fracture reduction among US women aged 45 years and older, 2001-2008. Journal of Bone and Mineral Research, v.26, n.3, p. 1116, 2011.

SILVA, M.S.; ANDRADE, S.R.S.; AMARAL, W.N. Fisiopatologia da osteoporose: uma revisão bibliográfica. FEMINA, v.43, n.6, p.241-244, 2015.

SMITH, E., SHAH, A. screening for Geriatric Syndromes: Falls, Urinary/Fecal Incontinence, and Osteoporosis. Clinics in Geriatric Medicine, v.34, n.1, p. 55-67, 2018.

SOUZA, M.P.G. Diagnóstico e tratamento da osteoporose. Revista Brasileira de Ortopedia, v.3, n.45, p.220-229, 2013.

TAVARES, J.H.; ALMEIDA, J.S.; MOURÃO, C.F.; MEIRA, R.; RIBEIRO, J. Avaliação qualitativa do tratamento da osteonecrose dos maxilares associada aos bisfosfonatos: aspectos atuais da literatura. Ciência Atual, v.8, n.2, p. 2$11,2016$.

TEMPONI, E.F.; JÚNIOR, L.H.C.; COSTA, L.P. Atypical femoral fracture due to chronic use of bisphosphonates: case report. Revista Brasileira de Ortopedia, v.50, n.4, p.482-485, 2015.

TORREGROSA, J.V.; RAMO, A.M. Uso de bisfosfonatos en la enfermedad renal crónica. Revista Nefrologia, v. 30, n. 3 , p. 288-296, 2010. 